\title{
Cancer Reports and Reviews
}

\section{Cancer liquid biopsy - A real advance of science}

\author{
Adrian Pablo Hunis* \\ Department of Internal Medicine, School of Medicine, University of Buenos Aires, Argentina
}

Twenty years ago I was optimistic about winning the fight against cancer, today my optimism is much more "moderate". According to epidemiological data, cancer mortality is declining by 2 to 3 percent per year, we have improved in almost all fields, diagnosis and treatment. I remain optimistic, but I do not visualize a cancer cure in the next 20 years. Possibly cease to be the leading cause of death in the Western world, since the therapeutic trend is to try to chronicle this disease (a fact that is only rarely achieved) but we can not prevent it.

Although we modify all the risk factors - tobacco, obesity, alcohol, inflammation, hereditary genes - cancer is still a natural phenomenon because of being alive. I do not think it is possible to prevent more than $40-50 \%$ of tumors. Therefore, they have to be diagnosed earlier. Focus on better detecting individual risk factors in the genome of each person and detecting cancer in much earlier stages, when it is beginning to develop and can still be cured.

The problem can be summed up, too, as if it were a mathematical equation. Each time a cell division occurs, there is a risk that an undetected error will occur and that genetic errors will accumulate. This certainty that the incidence of cancer will not decrease, is very worrying. The current figures are already very high and those that are glimpsed, are even more so. Not being able to avoid the development of a tumor, is not a failure. It is life itself. We are not designed to live 100 years. The numbers will increase because people live longer, and the main risk factor for cancer is AGE.

The incidence of cancer from 65 to 70 years increases exponentially. Since the 1970 s, $20 \%$ of the normal population have mutations in the blood, which are identical to those of cancer, which accumulate. One in two men and one in three women will have cancer. People live longer because they are diagnosed more cancers than before and because they die less from AIDS, cardiovascular diseases and other infectious diseases. There remains the cancer, which pathophysiologically, is a degenerative disease, and neurological conditions. We are facing two major threats. Alzheimer's disease and everything degenerative, and cancer.

\section{Advances: Liquid biopsy}

What is the Liquid biopsy? It is a blood test that detects mutations in cells. Cell clones with infiltrating and invasive properties, which leave their original organ, to nest in foreign tissues. These cell clones (metastases) may invade one or more organs. The most effective way to move to these cells is through the blood. But before actually colonizing, in the foreign tissue a handful of well-camouflaged cells invade the organ with mutated DNA. What makes the liquid biopsy is to intercept those "signaling" orders, which, through its study of molecular biology techniques, can make the diagnosis and follow the evolution of the tumor, in "real time".

\section{Advantages of Liquid biopsy}

- No more discomfort, pain and risk.

- The traditional biopsy consists of extracting a piece of the tumor by puncture or surgery. This tissue is then analyzed. It is a painful procedure. And it is not without risk, as is the case with mammograms and colonoscopies.

- They are not tests that can be repeated every few days. Something that does allow the liquid biopsy. And this makes it easier for us to see how cancer develops in near real-time, as we said earlier. In addition, the results can be known in less than 24 hours.

\section{Indications}

It allows to check if there are relapses before they are visible by the images (PET, TAC, RNM, etc.). The primary goal is to achieve early detection of cancer, when the hopes of healing are higher. The ideal situation is that this methodology can be generalized and applied in all types of tumors.

For now, liquid biopsy is being applied to lung, breast, colorectal, and melanoma cancers. Each cancer is different and evolves differently in each patient. There are tumors that present hundreds of mutations and can change almost from week to week. In one patient, for example, mutations may be different in primary cancer and in metastases. Current methods only allow us to see those changes when they are already widespread and affect hundreds of thousands or millions of cells. But the liquid biopsy quickly detects them as soon as a cell clone has changed its pattern. It is as if each cancer had a bar code and we passed it through the scanner in the supermarket box. This allows doctors not to waste precious time when making decisions. Have generalized and affect hundreds of thousands or millions of cells. But the liquid biopsy detects them quickly as soon as a cell clone has changed its pattern. It is as if each cancer had a bar code and we passed it through the scanner of the box of the supermarket. This allows doctors not to waste precious time when making decisions. A biopsy directly from a tumor gives information only on the DNA of that part of the tumor. The liquid biopsy in the blood increases the information and details. The liquid biopsy analyzes the blood for DNA residues, either of tumor cells that have died or living cells of a primary cancer that can cause new tumors (metastasis). Tumor cells shed DNA into the bloodstream.

Correspondence to: Adrian Pablo Hunis, M.D, Board Certified in Medical Oncology, Assistant Professor of Internal Medicine, School of Medicine, University of Buenos Aires, Head Professor of Oncology, Maimonides University, Argentina, E-mail: ahunis@fmed.uba.ar/aphunis@usa.net

Received: April 22, 2017; Accepted: May 10, 2017; Published: May 14, 2017 


\section{Methodology}

Once a blood sample is taken from the patient, DNA is isolated, eliminating red blood cells, platelets, and plasma. Experts take DNA from the nucleus of white blood cells. DNA is then read to look for tumor markers, that is, areas with a specific genetic component; The tumor cells differ clearly from the normal ones. There are already thousands of patients studied with Liquid biopsy, with more than $60 \%$ of the tumors, in early stages, a figure still low, but in a few years, when the method is standardized and more developed, the effectiveness will be greater than $90 \%$. In the world, there are half a dozen companies that are developing and adjusting the method, which will have a real test of early diagnosis of cancer, is expected by 2020 . This is very good news and just at that time, I'm sure I'll get my optimism back.

Copyright: $\odot 2017$ Hunis AP. This is an open-access article distributed under the terms of the Creative Commons Attribution License, which permits unrestricted use, distribution, and reproduction in any medium, provided the original author and source are credited. 